\title{
The crystal structure of gallic acid decarboxylase from Arxula adeninivorans
}

\author{
M Zeug ${ }^{1}$, N Markovic $^{2}$, C lancu $^{3}, \mathrm{~J} \mathrm{Tripp}^{4}$, M Oreb ${ }^{4}, \mathrm{~J} \mathrm{Choe}^{5}$ \\ ${ }^{1}$ East Carolina University, Greenville, NC, ${ }^{2}$ University of Illinois at Chicago College of Pharmacy, \\ Chicago, IL, ${ }^{3}$ East Carolina University, East Carolina Diabetes and Obesity Institute, Greenville, \\ NC, ${ }^{4}$ Goethe University Frankfurt, Frankfurt am Main, Germany, ${ }^{5}$ East Carolina University / East \\ Carolina Diabetes and Obesity Institute, Greenville, NC \\ matthias.zeug@gmx.de
}

Gallic acid decarboxylases (GADC), a protein found in many different types of bacteria and yeast, is an enzyme that decarboxylates gallic acid to form pyrogallol. Gallic Acid is a hydrolysis product of tannins. Tannins are plant metabolites that play a role in plant defense. They are known for their ability to precipitate proteins, being linked to different pathologies, including cancer. Gallic Acids are used in the pharmaceutical industries for their anticancer and antioxidant properties. The initial crystal of GADC in the space group P21212 diffracted at 2.0 Angstrom resolution and had nine molecules in the asymmetric unit. Structure determination by MAD failed, and there is no known homologs structure. A homologous protein (52\% amino acid identity) from Aspergillus niger was crystallized and diffracted to 1.8 Angstrom resolution, but the asymmetric unit had 18 molecules (in C2 space group). Another homologous protein (51\% amino acid identity) from Madurella mycetomatis also crystallized with only one molecule in the asymmetric unit (in P321 space group); this structure was determined by MAD phasing of the Se-Methione derivatized protein. Protein cofactors were found biochemically and validated in the crystal structure. Crystal structures revealed cobalt, potassium, and the substrate binding sites in a homotrimer. The potassium, required for activity, is at the center of the trimer, making trigonal prismatic coordination with OD and the carbonyl oxygen of Glu88 from each monomer. It positions the proximal and critical His86, part of a catalytic dyad, in the substrate binding site, through a twist in the connecting beta-strand. Cobalt further activates enzyme activity. Its binding site, formed by residues of two neighboring monomers, is removed from the active site, suggesting an allosteric mechanism of activation. The substrate analog 4-nitrocatechol is involved in a network of hydrogen bond interactions with W35, R39, T60, H86, Y150, and Q192. Mutation of any of these residues abolishes enzyme activity. The reaction is facilitated by His86, which is part of a catalytic dyad with Asp 40 .

Acta Cryst. (2020). A76, a177 